\title{
Route Reliability Modelling in Mobile ad hoc Network (R2M2) model using Bayesian algorithm
}

\author{
Pankaj Sharma ${ }^{1}$, Shruti Kohli ${ }^{2}$, Ashok K.Sinha ${ }^{3}$ \\ ${ }^{1 \& 3}$ Department of Information Technology, ABES Engineering College, Ghaziabad, UP, India \\ ${ }^{2}$ Department of Computer Science \& Engineering, BITEC, Noida, UP, India \\ ${ }^{1}$ sharma1pk@gmail.com; ${ }^{2}$ shruti@bitmesra.ac.in
}

\begin{abstract}
Mobile ad-hoc network technology has gained popularity in recent years by researchers on account of its flexibility, low cost and eases of deployment. The objective of proposed R2M 2 model is to evaluate the performance of MANET (Mobile ad hoc Network) operating with DSR( Dynamic Source Routing) as routing protocol. The R2M2 model for MANET is simulated and implemented using network simulator ns 2.34 and validated using Bayesian rules. The R2M2 model is able to find out the probability of having certain behavior (able to decide reliable or not reliable) of routes, in uncertain observations (not able to decide whether the route is reliable or not) in presence of drop and delay. For performance evaluation of MANET using the proposed R2M2 model input variables (like node density, number of active connections, duration of communication, node movement speed, pause time $\&$ data transfer rate etc. ) and output variables drop rate and delay have been taken as output variables. The R2M2 model helps in deciding the certain and uncertain behavior of routes and consequently the nodes and is found satisfactory.
\end{abstract}

\section{INTRODUCTION}

As the network is dynamic, the network topology continuously experiences alterations during deployment. The biggest challenge in MANETs is to find a route between communicating nodes and behavior of nodes forming the route, some of them are cooperating and some are non-cooperating or selfish nodes. A selfish node is a node that wants to save battery life for its own communication can endanger the correct network operation by simply not participating to the routing protocol or by not executing the packet forwarding (this attack is also known as the black hole attack). Current ad hoc routing protocols cannot cope with the selfishness problem and network performances severely degrade.

In this paper performance of MANET is evaluated by implementing Dynamic Source Routing (DSR) protocol under different scenarios. DSR is an On-demand source routing 
protocol. In DSR the route routes are discovered after source sends a packet to a destination node in the ad-hoc network. The source node initially does not have a route to the destination when the first packet is sent. The DSR has two functions first is route discovery and the second is route maintenance $[1,2]$.

\section{ROUTING IN MANET}

A MANET routing algorithm should not only be capable of finding the shortest route between the source and destination, but it should also be adaptive, in terms of the changing state of the nodes, the changing load conditions of the network and the changing state of the environment. MANET routing algorithms can be classified into three categories as proactive, reactive or hybrid [3]. Proactive algorithms try to maintain up-to-date routes between all pairs of nodes in the network at all times. Examples of proactive algorithms are DestinationSequence Distance- Vector routing (DSDV) and Optimized Link State Routing (OLSR) [4]. Reactive algorithms only maintain routing information that is strictly necessary: they set up routes on demand when a new communication session is started, or when a running communication session falls without route. Examples of reactive routing algorithms include Dynamic Source Routing (DSR) and Adhoc On-demand Distance-Vector routing (AODV) [5].

"Routing is the process of information exchange from one host to the other host in a network."[6]. Routing is the mechanism of forwarding packet towards its destination using most efficient route. Efficiency of the route is measured in various metrics like, Number of hops, traffic, security, etc. In Ad-hoc network each host node acts as specialized router itself [7].

Routing protocol for ad-hoc network can be categorized in three strategies.

a) Flat Vs Hierarchical architecture.

b) Pro- active Vs Re- active routing protocol.

c) Hybrid protocols.

Hierarchical network architecture topology consists of multiple layers where top layers are more seen as master of their lower layer nodes. There are cluster of nodes and one gateway node among all clusters has a duty to communicate with the gateway node in other cluster. In this schema there is a clear distribution of task. Burden of storage of network topology is on gateway nodes, where communicating different control message is dependent on cluster nodes.

But this architecture breaks down when there is single node failure (Gateway node). Gateway nodes become very critical for successful operation of network. Examples include Zone-based Hierarchical Link State (ZHLS) routing protocol [8]. Where in flat architecture there is no layering of responsibility. Each and every node does follow the same routing algorithm as any other node in the network. 
In proactive routing scheme every node continuously maintains complete routing information of the network. This is achieved by flooding network periodically with network status information to find out any possible change in network topology.

Current routing protocol like Link State Routing (LSR) protocol (open shortest route first) and the Distance Vector Routing Protocol (Bellman-Ford algorithm) are not suitable to be used in mobile environment.

Destination Sequenced Distance Vector Routing protocol (DSDV) and Wireless routing protocols were proposed to eliminate counting to infinity and looping problems of the distributed Bellman-Ford Algorithm.

Examples of Proactive Routing Protocols are: [9].
a) Global State Routing (GSR).
b) Hierarchical State Routing (HSR).
c) Destination Sequenced Distance Vector Routing (DSDV).

Every node in this routing protocol maintains information of only active routes to the destination nodes. A route search is needed for every new destination therefore the communication overhead is reduced at the expense of delay to search the route. Rapidly changing wireless network topology may break active route and cause subsequent route search [8].

Examples of reactive protocols are:
a) Ad hoc On-demand Distance Vector Routing (AODV).
b) Dynamic Source Routing (DSR).
c) Location Aided Routing (LAR).
d) Temporally Ordered Routing Algorithm (TORA).

There exist a number of routing protocols of globally reactive and locally proactive states. Hybrid routing algorithm is ideal for Zone Based Routing Protocol (ZRP) [8][9].

\section{DSR (DYNAMIC SOURCE ROUTING)}

This is an On-demand source routing protocol. In DSR the route routes are discovered after source sends a packet to a destination node in the ad-hoc network. The source node initially does not have a route to the destination when the first packet is sent. The DSR has two functions first is route discovery and the second is route maintenance $[10,11]$.

\section{STEPS TO IMPLEMENT THE PROPOSED R2M2 MODEL}

The algorithm implemented in this paper follows the steps:

1. Define the MANET with state input variables

2. Determine the scenarios using state variables 
3. Simulate the behavior of MANET using scenarios in step (2)

4. Find out all possible routes

5. Compute the performance of route by considering the performance of each nodes participating in the route

6. Filter out behavior of routes based on result computed in step (6)

7. Validate the R2M2 model with bayes 'probability test

\section{TOOLS \& METHODOLOGY USED IN SIMULATIONS}

In this paper we have used various tools such as network simulator version 2.34 (NS2.34) for getting the simulation results by writing and running the TCL script, applying the parameters in Table1, in addition we have taken the help of traffic generation tool such as cbrgen.tcl and mobile movement scenario generation tool such as Bonmotion 1.4, after getting the results.

For implementation DSR protocol in MANET environment, we prepared a scenario as shown in Table 1 and initial model of MANET is shown in fig. 1 which shows some input variables and output variable described as following:

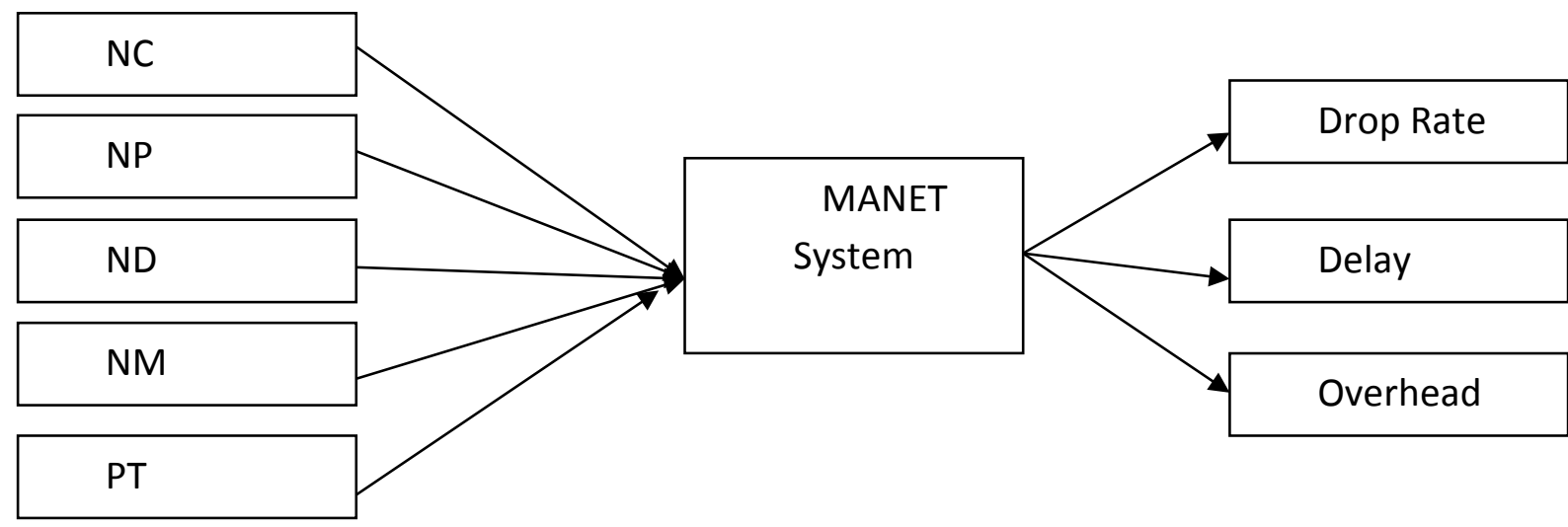

Fig. 1 Block Diagram of MANET system

Where NC: number of connections, PT: Pause time, ND:Node density, NM:Node mobility

PDF: Packet Delivery Fraction, NRL: Normalized Routing Load,AE2ED: Average End 2 End Delay. 
Table 1. Scenario parameters

\begin{tabular}{|l|l|}
\hline \multicolumn{2}{|c|}{ Simulation Parameters } \\
\hline Routing Protocol & DSR \\
\hline Mobility Model & RPGM \\
\hline Simulation Time & 10 \\
\hline Number of Nodes & $5,10,15,20,25$ \\
\hline Simulation Area & $\mathrm{x}=1000 \mathrm{~m}, \mathrm{y}=1000 \mathrm{~m}$ \\
\hline Speed & $5 \mathrm{~m} / \mathrm{sec}$ \\
\hline Pause Time & 5 \\
\hline Traffic Type & CBR \\
\hline Packet Size & 512 bytes \\
\hline Rate & 5 packets/sec \\
\hline Number of Connections & $3,7,10,15,18$ \\
\hline Seed & 1.0 \\
\hline
\end{tabular}

A scenario defined in Table1 is simulated with network simulator 2.34 and output is generated. Having analyzed the results, routes are computed and performance of routes is evaluated as dropped packet rate which is indirectly proportional to packet delivery ratio and delay in packet transmission, sample route performance is shown in Table 2.

Table 2. Occurrences of route1 with drop and delays

\begin{tabular}{|c|l|l|}
\hline Possible occurrences of route1 & Drop & Delay \\
\hline 1 & 0 & 14.57 \\
\hline 2 & 0 & 14.85 \\
\hline 3 & 34 & 9.53 \\
\hline 4 & 36 & 9.53 \\
\hline 5 & 0 & 8.57 \\
\hline 6 & 23 & 13.85 \\
\hline 7 & 29 & 9.53 \\
\hline 8 & 0 & 8.53 \\
\hline
\end{tabular}

\section{PROPOSED R2M2 MODEL}

The model is tested and validated on route 1 shown in table 2 . Table 2 is obtained after simulating the scenario defined in table 1 using network simulator. Hence with respect to the results shown in table 2 , the population size of all possible outcomes of routes is 40 and out of which 65\% (26) unique outcomes of routes are observed; assuming that unique outcomes are taken as sample space for the proposed model. In this paper we have randomly chosen a route 
(route 1) for testing the behavior. The total occurrences of route 1 in sample space $30 \%$ (8) times having different behavior i.e in some occurrences it drops packets and in some occurrences it does not drop. The objective of this paper is to predict the behaviors of route1, whether the route's behavior is certain or uncertain. For testing and validating the behavior, some observations of route 1 have been taken with respect to drop and delay. Out of total observations, in $50 \%$ cases it drops packets and in remaining $50 \%$ it does not drop. If we cannot say that the drop is the only parameter for prediction of behavior of any route, and then based on the drop, it is difficult to determine the actual behavior of route. In presence of delay it may behave differently. For validating the behavior of route we have taken another parameter that is delay. In this paper initially we have chosen the threshold value of delay is $10 \mathrm{~ms}$; although in realistic it may be very low.

Table 3: Behavior prediction table

\begin{tabular}{|c|c|c|c|}
\hline Route1 (1-0-2) & Delay $>=10$ & Delay $<10$ & $\begin{array}{l}\quad \text { Marginal } \\
\text { probability } \\
\text { (Total } \\
\text { Probability) }\end{array}$ \\
\hline \multirow[t]{2}{*}{$\begin{array}{l}\text { Case-1 } \\
\text { (Packets dropping) }\end{array}$} & 1 & 3 & 04 \\
\hline & $1 / 4=25 \%$ & $03 / 4=75 \%$ & $4 / 8=50 \%$ \\
\hline $\begin{array}{c}\text { Case- } 2 \\
\text { (Packets not dropping) }\end{array}$ & 2 & 2 & 04 \\
\hline Marginal probability & $02 / 04=50 \%$ & $2 / 04=50 \%$ & $4 / 8=50 \%$ \\
\hline
\end{tabular}

A route in a network may comprise of two or more nodes and the behavior of a route depends upon the node's behavior and transmission medium. In this paper we primarily will focus only on node's behavior and later on transmission processing (delay). Initially after analyzing the behavior based up on the simulation output) we can categorise the node behavior in Mobile ad hoc network environment in two categories either having certain behavior (the nodes which do not loss the information) or having uncertain behavior (which may loss information).

The performance of MANET under operation of routing protocol depends upon the behavior of routes. In this paper the MANET is simulated under DSR (Dynamic Source Routing) protocol. The performance of MANET depends on delay and packet drop rate which effect the behavior of route, If we assume that the processing time of the packets in network is $10 \mathrm{~ms}$. thus after classifying the outcomes of the route including the delay parameter also, one category of outcomes is having delay $>=10 \mathrm{~ms}$ and another category is having delay $<10 \mathrm{~ms}$. Both category is under consideration of zero drop rate i.e. $100 \%$ packet delivery ratio, the categorization is shown in Table 3.

This paper is focusing the behavior of a route (1-0-2) that has both type of behavior In MANET system, based on the scenarios defined in table1, it has been observed that in $50 \%$ 
cases the route is having certain behavior, in case of either dropping minimum data packets or zero data packets and in rest of the $50 \%$ cases the observed route is having uncertain behavior, which means the route is dropping packets.

For validating the issues in deciding the actual behavior of route either certain or uncertain, the outcomes of the route are passed to Bayesian decision theorem; the base equation of bayes theorem is[12][13] :

$$
\mathbf{P}(\mathbf{A} \mid \mathbf{B})=\frac{\mathbf{P}(\mathbf{A}) \cdot \mathbf{P}(\mathbf{B} \mid \mathbf{A})}{[\mathbf{P}(\mathbf{A}) \cdot \mathbf{P}(\mathbf{B} \mid \mathbf{A})]+\left[\mathbf{P}\left(\mathbf{A}^{\prime}\right) \cdot \mathbf{P}\left(\mathbf{B} \mid \mathbf{A}^{\prime}\right)\right]}
$$

Where event $A$ is the hypothesis and event $B$ is the evidence.

Let $\mathrm{X}$ denotes certain behavior (not dropping packets) and $\mathrm{X}^{\prime}$ denotes the uncertain behavior (dropping packets), $D$ is the case where delay $>=10 \mathrm{~ms}$ and $D^{\prime}$ is the case where delay is $<10 \mathrm{~ms}$. In this proposed model we need to test the following observations:

Prior assumption is that $50 \%$ of the total cases are having certain behavior so prior probability of randomly selecting a route and having certain behavior is $P(X)=0.5$. The goal is to find the probability that selected route is having certain behavior based on the observations shown in table 3 ; where $50 \%$ of positive cases of a route (positive cases are those having drop rate zero) having delay $>=10 \mathrm{~ms}, 50 \%$ of positive cases having delay $<10 \mathrm{~ms}, 25 \%$ of negative cases (negative cases which drop the packets) having delay $>=10 \mathrm{~ms}$ and $75 \%$ of negative cases having delay $<10 \mathrm{~ms}$. We can say that $P(X)=.5$ (Since $50 \%$ of routes are having certain behavior), $P\left(X^{\prime}\right)=.5$ (Since $65 \%$ of routes are having uncertain behavior), $P(D \mid X)=.5$ and $P\left(D \mid X^{\prime}\right)=.25$.

If we compare the above scenario with the realistic scenario where delay should be minimum, assuming. For validating that the cases where route does not drop packet and delay in transmission is greater than $10 \mathrm{~ms}$, then for testing the behavior of route with the following equation (2):

$$
\mathbf{P}(\mathbf{X} \mid \mathbf{D})=\frac{\mathbf{P}(\mathbf{X}) \cdot \mathbf{P}(\mathbf{D} \mid \mathbf{X})}{[\mathbf{P}(\mathbf{X}) \cdot \mathbf{P}(\mathbf{D} \mid \mathbf{X})]+\left[\mathbf{P}\left(\mathbf{X}^{\prime}\right) \cdot \mathbf{P}\left(\mathbf{D} \mid \mathbf{X}^{\prime}\right)\right]}
$$

Now it has been observed that $66.6 \%$ results indicate for a route to be having certain behavior and $33.4 \%$ indicates for a route to be having uncertain behavior. For validating that the cases where route does not drop packet and delay in transmission is less than $10 \mathrm{~ms}$, then for testing the behavior of route with the following equation (2):

Where $P(X)=.5 \quad$ (Since $50 \%$ of routes are certain), $P\left(X^{\prime}\right)=.5$ (Since $65 \%$ of routes are uncertain), $P\left(D^{\prime} \mid X\right) \quad=.5$ and $P\left(D^{\prime} \mid X^{\prime}\right)=.75$. The following computation will help to find out the probability of the positive cases having delay $<10 \mathrm{~ms}$

$$
\mathbf{P}\left(\mathbf{X} \mid \mathbf{D}^{\prime}\right)=\frac{\mathbf{P}(\mathbf{X}) \cdot \mathbf{P}(\mathbf{D} \prime \mid \mathbf{X})}{[\mathbf{P}(\mathbf{X}) \cdot \mathbf{P}(\mathbf{D} / \mid \mathbf{X})]+\left[\mathbf{P}\left(\mathbf{X}^{\prime}\right) \cdot \mathbf{P}\left(\mathbf{D} / \mid \mathbf{X}^{\prime}\right)\right]}
$$

Finally it has been observed that in $40 \%$ cases the behavior of route is satisfactory and in $60 \%$ the behavior is unsatisfactory. 
Table 4 Behavior of route1 observed after validation

\begin{tabular}{|c|c|}
\hline Behavior of route & probability \\
\hline $\begin{array}{c}\text { Probability of having certain behavior of positive } \\
\text { cases of route when delay is high ( }>=10 \mathrm{~ms})\end{array}$ & $66.6 \%$ \\
\hline $\begin{array}{c}\text { Probability of having certain behavior of negative } \\
\text { cases of route when delay is low ( }<10 \mathrm{~ms})\end{array}$ & $40 \%$ \\
\hline $\begin{array}{c}\text { Probability of having uncertain behavior of } \\
\text { negative cases of route when delay is low }(<10 \mathrm{~ms})\end{array}$ & $60 \%$ \\
\hline $\begin{array}{c}\text { Probability of having uncertain behavior of positive } \\
\text { cases of route when delay is low }>=10 \mathrm{~ms}\end{array}$ & $33.4 \%$ \\
\hline
\end{tabular}

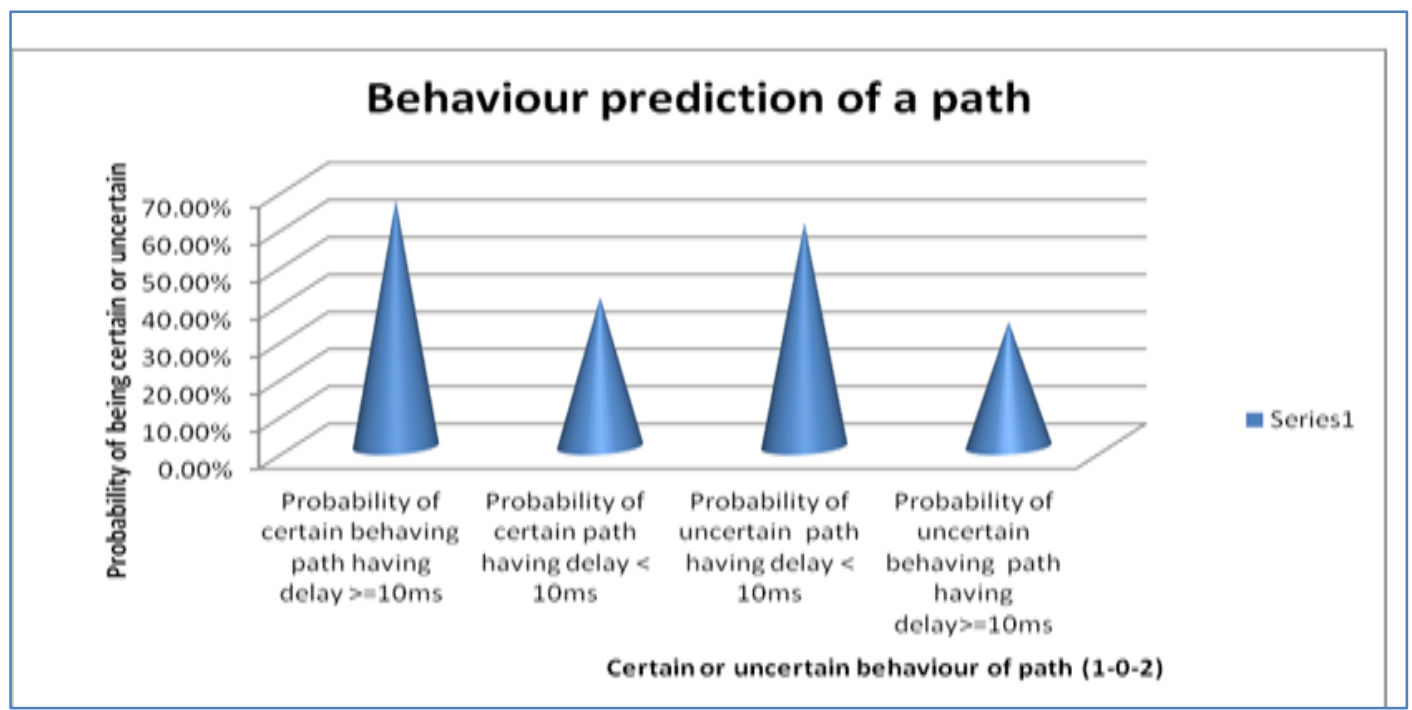

Fig. 2 : Validation Results comparision of occurance of rout 1

\section{CONCLUSION}

It has been concluded that the model is found satisfactory for reliability testing of a route. Figure 2 shows the graphical view of behavior analysis of route 1 and R2M2 model can be applied to any number of routes for behavior prediction. The model is able to find out that the probability of chosen route is 0.66 which indicates for a route to be having certain behavior and with probability .33 shows a route to be having uncertain behavior, while delay is greater than $10 \mathrm{~ms}$.

The probability of the cases, where route does not drop packet and delay in transmission is less than $10 \mathrm{~ms}$, it has been observed that with probability .40 , it shows the behavior of route is reliable and with probability .60 the route is not reliable. Thus according to MAP (Maximum Posterior) the route will belongs to the class of reliability. The outcome of model is shown in table 4. 


\section{REFERENCES}

[1]. Loutfi, Valerie, Bruno. "Securing mobile adhoc networks", MP71 project, 2003

[2]. Sergio Marti, T. J. Giuli, Kevin Lai, Mary Baker "Mitigating Routing Misbehavior in Mobile Ad Hoc Networks", Proceedings of the 6th annual international conference on Mobile computing and networking, Boston, Massachusetts, 2000,Pages: $255-265$

[3]. E.M. Royer and C.K. Toh. A review of current routing protocols for ad hoc mobile wireless networks. In IEEE Personal Communications, volume 6, April 1999.

[4]. T. Clausen and P. Jacquet. "Optimized link state routing protocol (olsr)". RFC 3626: Optimized link state routing protocol (OLSR), Oct 2003.

[5]. C. Perkins. "Ad hoc on-demand distance vector routing. Internet-Draft", draft-ietf-manet-aodv-00.txt, November 1997.

[6]. Humayun Bakht, "Computing Unplugged, Wireless infrastructure, Some Applications of Mobile ad hoc networks", http://www.computingunplugged.com/issues/ issue200410/00001395001.html, April2003.

[7]. Charles E.Perkins and Elizabeth M. Royer, "Ad hoc on demand distance vector (AODV) routing (Internet-Draft)", Aug-1998.

[8]. Mario Joa-Ng, "A Peer-to-Peer Zone-Based Two-Level Link State Routing for Mobile Ad Hoc Networks", IEEE Journal on selected areas in communications, Vol. 17, No. 8, Aug-1999.

[9]. Padmini Misra, "Routing Protocols for ad hoc mobile wireless Networks", http://www.cse.ohiostate.edu / jain/cis788-99/ftp/adhoc_routing/\#TDRP, Nov-1999.

[10]. Loutfi, Valerie, Bruno. "Securing mobile adhoc networks", MP71 project, 2003

[11]. Sergio Marti, T. J. Giuli, Kevin Lai, Mary Baker "Mitigating Routing Misbehavior in Mobile Ad Hoc Networks", Proceedings of the 6th annual international conference on Mobile computing and networking, Boston, Massachusetts, 2000,Pages: 255-265.

[12]. Popper, K. (1959). The Logic of Scientific Discovery. Routledge, London, New York. St.Andrews (2003). Bayes. School of Mathematics and Statistics, University of St Andrews Scotland, http://wwwgap.dcs.st-and.ac.uk/ history/Mathematicians/Bayes.html. Edited by John O'Connor and Edmund Robertson.

[13]. UCalgary (2003). Bayes Theorem. University of Calgary, Department of Mathematics and Statistics, Division of Statistics and Actuarial Science, http://balducci.math.ucalgary.ca/. 